\title{
Sorting fission from parasitic coincidences of neutrons and gamma rays in plastic scintillators using particle times of flight
}

\author{
V. Bottau a*, C. Carasco ${ }^{\mathrm{a}}$, B. Perot ${ }^{\mathrm{a}}$, C. Eleon ${ }^{\mathrm{a}}$, R. De Stefano ${ }^{\mathrm{a}}$, L. Isnel ${ }^{\mathrm{a}}$, I. Tsekhanovich $^{\mathrm{b}}$ \\ (a) CEA, DES, IRESNE, DTN, SMTA, Nuclear Measurement Laboratory, Cadarache, F-13108 Saint-Paul-Lez-Durance, France \\ (b) Université de Bordeaux, CNRS, CENBG, UMR 5797, F-33170 Gradignan, France \\ (*) corresponding author: vincent.bottau@ cea.fr
}

\begin{abstract}
This work addresses the use of plastic scintillators as an alternative to ${ }^{3} \mathrm{He}$ detectors for radioactive waste drum characterization. The time response of scintillators is three orders of magnitude faster than that of gas proportional counters and they offer similar neutron detection efficiency at lower cost. However, they are sensitive to gamma rays and the commonly used Pulse Shape Discrimination technique is not possible with basic PVT scintillators. This paper reports on an innovative data processing technique allowing to extract spontaneous fission events from parasitic coincidences, such as those from the $(\alpha, n)$ reactions accompanied by correlated gamma rays or from pure gamma-ray sources emitting correlated radiations. The proposed approach makes advantage of differences in the pulse detection times recorded in measurements with the ${ }^{252} \mathrm{Cf}$, AmBe and ${ }^{60} \mathrm{Co}$ sources. More precisely, a 2D histogram of time delays between the detected $2^{\text {nd }}$ and $1^{\text {st }}$ pulses, on the $x$-axis, and between the $3^{\text {rd }}$ and $2^{\text {nd }}$ pulses, on the $y$-axis, is found to allow for selection of a region of interest most relevant to spontaneous fission events.
\end{abstract}

Keywords - Plastic PVT scintillators, spontaneous fission prompt neutrons and gamma rays, coincidence analysis, radioactive waste drums.

\section{INTRODUCTION}

$I^{N}$ the context of plutonium bearing waste drum characterization by passive neutron coincidence counting, conventional measurement collars based on ${ }^{3} \mathrm{He}$ gas counters permit a temporal discrimination between spontaneous fission neutrons and parasitic neutrons from $(\alpha, n)$ reactions [1]. The ${ }^{3} \mathrm{He}$ proportional counters are well suited for neutron multiplicity counting because of their large capture cross section for thermal neutrons, leading to excellent detection efficiency, and their insensitivity to gamma rays. However, the shortage of ${ }^{3} \mathrm{He}$ gas has greatly increased the cost of these detectors, leading to a worldwide effort to find possible alternatives, especially in the field of homeland security and safeguards [2], [3]. The Nuclear Measurements Laboratory of CEA Cadarache (France) makes studies of lowcost EJ-200 Polyvinyl Toluene (PVT) plastic scintillators as a potential alternative for neutron coincidence collars [4], [5]. Efficient neutron detection with ${ }^{3} \mathrm{He}$ counters requires neutron thermalization, which lasts several tens of microseconds, whereas plastic proton recoil detectors are directly sensitive to fast neutrons, which need just a few tens of nanoseconds to cover the source-detector distance. Therefore, the coincidence window can be three orders of magnitude shorter with PVT scintillators than with ${ }^{3} \mathrm{He}$ counters, which limits dramatically accidental coincidences.

However, plastic scintillators are strongly sensitive to gamma rays, which, in addition, may scatter between detectors and thus produce parasitic coincidences that will be merged with useful (i.e., different-particle) coincidence events expected from fission. For instance, $(\alpha, n)$ reactions accompanied by correlated gamma rays, or pure gamma ray sources emitting correlated gamma radiations can induce undesired high-order coincidences [6] due to the mentioned multi-detector hit events by a single gamma-ray or neutron, that are currently under worldwide investigations [4], [7-10]. Although some plastic scintillators allow via the Pulse Shape Discrimination (PSD) to sort neutron and gamma ray events [11-14], they are not a practical alternative for radioactive waste drum characterization. Indeed, a large detection efficiency is needed for coincidence measurements, but the PSD figure of merit (FOM) decreases for large-size scintillators due to multiple light scattering and lower photoelectron statistics [15-18]. In addition, the FOM also decreases at intermediate and highcount rates due to the pile-up effect-expected to be present for intermediate and high-level radioactive wastes. A possible solution would be to use a large number of small size PSD scintillators (typically less than 5 " $\times 5$ ") but more than 100 detectors would be needed to fully cover the detection solid angle, even for limited-in-size radioactive waste packages like $120 \mathrm{~L}$ drums $(\varnothing \approx 44 \mathrm{~cm}, \mathrm{~h} \approx 80 \mathrm{~cm})$. The cost and complexity of the measurement system, with its electronics, would not be advantageous with respect to ${ }^{3} \mathrm{He}$ detectors.

To overcome the above limitations, we have developed a method exploiting the differences in the time-of-flight of neutrons and gamma rays detected in triple coincidences with an array of PVT detectors. A 2D histogram of the time delays between the detected $2^{\text {nd }}$ and $1^{\text {st }}$ pulses, on the $\mathrm{x}$-axis, and between the $3^{\text {rd }}$ and $2^{\text {nd }}$ pulses, on the $y$-axis, is found to allow for selection of a region of interest most relevant to spontaneous fission events. This papers reports the very first tests performed with bare radioactive sources measured separately to highlight the region of interest of the useful signal $\left({ }^{252} \mathrm{Cf}\right.$ spontaneous fission source) with respect to different sources of background 
noise $\left({ }^{60} \mathrm{Co}\right.$ and $\left.\mathrm{AmBe}\right)$. The ${ }^{252} \mathrm{Cf}$ source emits correlated fission prompt neutrons and gamma rays, ${ }^{60} \mathrm{Co}$ emits correlated 1.173 and $1.332 \mathrm{MeV}$ gamma rays and $\mathrm{AmBe}$ emits a single neutron correlated with a $4.439 \mathrm{MeV}$ gamma ray following the ${ }^{9} \mathrm{Be}(\alpha, n){ }^{12} \mathrm{C}^{*}$ reaction. The multiplicity analysis with the traditional shift register method is also reported to allow a comparison with the new approach.

\section{EXPERIMENTAL SETUP}

The experimental setup consists of eight EJ-200 (Eljen Technology) $10 \times 10 \times 100 \mathrm{~cm}^{3}$ plastic scintillators coupled to 3M-P-E1-X-N (ET Enterprises Ltd.) photomultipliers. These detectors assembled by Scionix are shown in Fig. 1. Calibrations sources are in central position, at $35 \mathrm{~cm}$ from each detector considering the orthogonal direction, and surrounded by $2 \mathrm{~mm}$ of lead, mainly to cut the $59 \mathrm{keV}$ gamma rays of ${ }^{241} \mathrm{Am}$ in the $3.68 \mathrm{GBq} \mathrm{AmBe}$ source. The 8-channel DT5730B digitizer (CAEN S.p.A), used with the built-in DPP-PSD firmware, samples the analog pulse trains with a $500 \mathrm{MHz}$ frequency on 14 bytes [19] and delivers a list of the pulse time stamps calculated by a Constant Fraction Discriminator algorithm (CFD) to the acquisition computer through a maximum $50 \mathrm{MB} / \mathrm{s}$ USB2 connector. The v1.0.4 CoMPASS software from CAEN drives acquisition [20], in which the long gate for charge integration and the record length (size of the acquisition window, i.e. number of samples) have been set to $60 \mathrm{~ns}$ and $182 \mathrm{~ns}$, respectively. A low-energy threshold of $100 \mathrm{keVee}$ (electron equivalent energy) is also set in order to filter the intense $59 \mathrm{keV}$ line from AmBe. In practice, this threshold will always be useful for plutonium characterization in radioactive wastes because of a plurality of intense gamma and X-ray lines from plutonium isotopes and ${ }^{241} \mathrm{Am}[6,21]$.

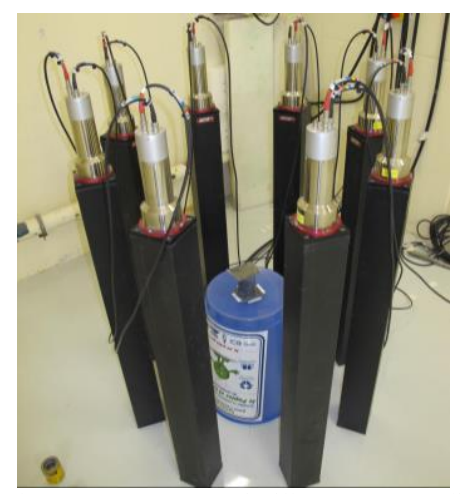

Fig. 1. Experimental set-up: 8 EJ-200 scintillators surrounding the ${ }^{252} \mathrm{Cf}$ source placed on a $50 \mathrm{~cm}$ height support.

\section{DATA ANALYSIS}

\section{A. Shift Register method}

The data acquired with ${ }^{252} \mathrm{Cf}$, AmBe and ${ }^{60} \mathrm{Co}$ sources are processed with CERN's ROOT software [22] based on the Rossi-Alpha distribution and on the historical shift register method for the determination of number of fired detectors, which was originally developed for neutron coincidence collars using ${ }^{3} \mathrm{He}$ counters [1], [23]. The Rossi-Alpha distribution (detection times of next pulses after each detected pulse) obtained with the ${ }^{252} \mathrm{Cf}$ source is given in Fig. 2. Two main contributions are visible, first $\gamma \gamma$ pairs with time intervals below $10 \mathrm{~ns}$, then $\gamma \mathrm{n}$ pairs with time delays in the (10-50 ns) range.

For the shift register application, a $100 \mathrm{~ns}$ coincidence window is used to integrate real coincidences. The basics of the shift register method are illustrated in Fig. 3. The "Real + Accidental" $(\mathrm{R}+\mathrm{A})$ register accounts for all types of coincidences, while "Accidental" (A) records only random coincidences, $(\mathrm{R}+\mathrm{A})$ and $(\mathrm{A})$ windows being separated by $1 \mu \mathrm{s}$. The "M0" multiplicity corresponds to the absence of coincidence in the 100 ns window opened by a neutron or gamma pulse (no pulse in the other detectors), M1 records coincidences with another pulse (i.e. a pair), etc.

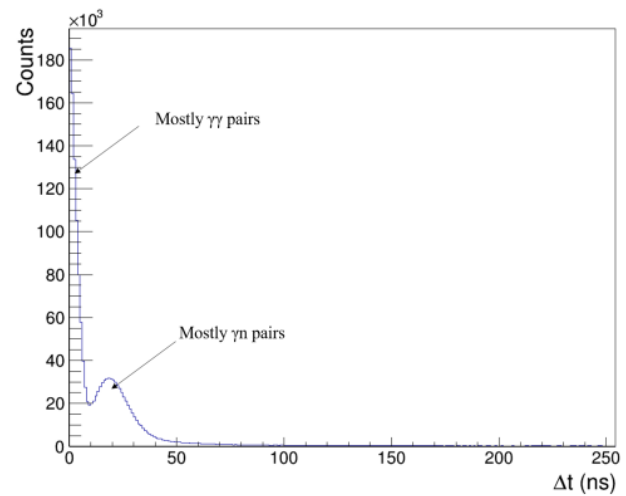

Fig. 2. Rossi-Alpha distribution obtained for the measurement in presence of the ${ }^{252} \mathrm{Cf}$ source

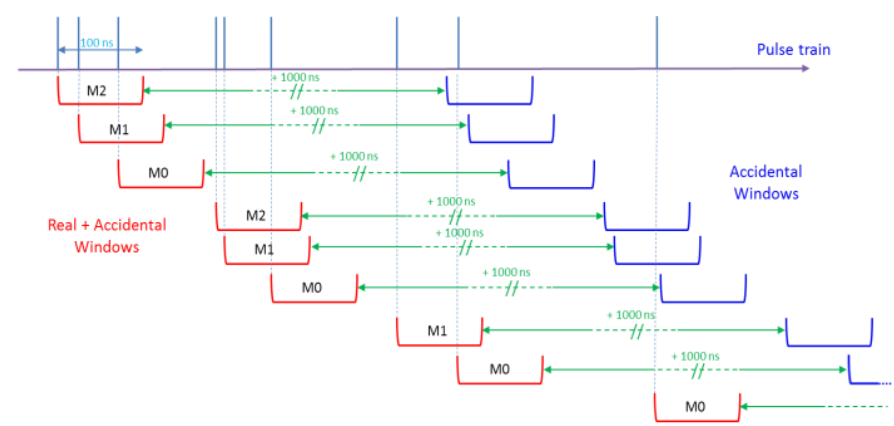

Fig. 3. Diagram of the shift register method, see text for details.

Table I and Table II report 5 min acquisition data of the background noise and of the individual radioactive sources measured separately.

TABLE I

NUMBER OF EVENTS DETECTED FOR DIFFERENT MULTIPLICITIES, ALONG WITH THE COUNT RATES OF SINGLES, DOUBLES, AND TRIPLES (S, D AND T, RESPECTIVELY) DURING BACKGROUND NOISE MEASUREMENT

\begin{tabular}{ccc}
\hline \hline & \multicolumn{2}{c}{ Background noise } \\
\cline { 2 - 3 } & $\mathrm{R}+\mathrm{A}$ & $\mathrm{A}$ \\
\hline M0 & $2259505 \pm 1503$ & $2349237 \pm 1533$ \\
M1 & $89387 \pm 299$ & $1860 \pm 43$ \\
M2 & $2066 \pm 45$ & $64 \pm 8$ \\
M3 & $130 \pm 11$ & $2 \pm 1$ \\
M4 & $46 \pm 7$ & $1 \pm 0$ \\
M5 & $20 \pm 4$ & $0 \pm 0$ \\
M6 & $8 \pm 3$ & $0 \pm 0$ \\
M7 & $2 \pm 1$ & $0 \pm 0$ \\
S & \multicolumn{3}{c}{$7837 \mathrm{~s}^{-1}$} \\
D & $308 \mathrm{~s}^{-1}$ \\
T & \multicolumn{3}{c}{$10 \mathrm{~s}^{-1}$}
\end{tabular}


TABLE II

NUMBER OF EVENTS DETECTED FOR DIFFERENT MULTIPLICITIES, (AFTER SUBTRACTION OF THE BACKGROUND NOISE) ALONG WITH THE COUNT RATES OF SINGLES, DOUBLES, AND TRIPLES (S, D AND T, RESPECTIVELY)

\begin{tabular}{lrrrrrr}
\hline \hline & \multicolumn{2}{c}{${ }^{252} \mathrm{Cf}(288 \mathrm{kBq})$} & \multicolumn{2}{c}{ AmBe $(3.68 \mathrm{GBq})$} & \multicolumn{2}{c}{${ }^{60} \mathrm{Co}(882 \mathrm{kBq})$} \\
\cline { 2 - 7 } & \multicolumn{1}{c}{$\mathrm{R}+\mathrm{A}$} & \multicolumn{1}{c}{$\mathrm{A}+\mathrm{A}$} & \multicolumn{1}{c}{$\mathrm{A}+\mathrm{A}$} & \multicolumn{1}{c}{$\mathrm{A}$} \\
\hline M0 & 3379137 & 4479459 & 15577017 & 16671802 & 53638186 & 57771864 \\
& \pm 2810 & \pm 3029 & \pm 4483 & \pm 4623 & \pm 7626 & \pm 7904 \\
M1 & 857974 & 8310 & 1141198 & 102194 & 4947762 & 989025 \\
& \pm 1018 & \pm 110 & \pm 1149 & \pm 325 & \pm 2264 & \pm 996 \\
M2 & 209237 & 1296 & 59474 & 5790 & 251249 & 81991 \\
& \pm 462 & \pm 38 & \pm 252 & \pm 77 & \pm 505 & \pm 287 \\
M3 & 38098 & 257 & 2312 & 253 & 9079 & 3516 \\
& \pm 196 & \pm 16 & \pm 51 & \pm 16 & \pm 97 & \pm 59 \\
M4 & $4806 \pm 70$ & $42 \pm 7$ & $67 \pm 13$ & $14 \pm 4$ & $239 \pm 18$ & $97 \pm 10$ \\
M5 & $401 \pm 21$ & $5 \pm 2$ & $-9 \pm 6$ & $0 \pm 0$ & $-11 \pm 5$ & $2 \pm 1$ \\
M6 & $18 \pm 6$ & $1 \pm 1$ & $-3 \pm 4$ & $0 \pm 0$ & $-7 \pm 3$ & $0 \pm 0$ \\
M7 & $-1 \pm 2$ & $0 \pm 0$ & $-2 \pm 1$ & $1 \pm 0$ & $-2 \pm 1$ & $0 \pm 0$ \\
\hline S & & $14966 \mathrm{~s}^{-1}$ & & $55934 \mathrm{~s}^{-1}$ & & $196155 \mathrm{~s}^{-1}$ \\
D & $4668 \mathrm{~s}^{-1}$ & & $3843 \mathrm{~s}^{-1}$ & & $14381 \mathrm{~s}^{-1}$ \\
T & $1175 \mathrm{~s}^{-1}$ & & $175 \mathrm{~s}^{-1}$ & & $342 \mathrm{~s}^{-1}$
\end{tabular}

The count rates of singles ( $\mathrm{S}$, sum of all the triggers), doubles (D, which is $\mathrm{S}$ times the difference between the mean values of $(\mathrm{R}+\mathrm{A})$ and $(\mathrm{A})$ registers of $\mathrm{M} 1)$ and triples (T, which is a complex deconvolution of $(\mathrm{R}+\mathrm{A})$ and $(\mathrm{A})$ distributions with the registers M2, M3, M4, etc.) are calculated following the equations detailed in [23-25]. As follows from (2-4), the signalto-noise ratio (SNR) increases significantly from singles to triples because M1 (two fired detectors) is dominated by correlated $\gamma \mathrm{n}$ pairs of $\mathrm{AmBe}$ and $\gamma \gamma$ pairs of ${ }^{60} \mathrm{Co}$, whereas higher-order multiplicities (M2, M3, etc.) are dominated by ${ }^{252} \mathrm{Cf}$ spontaneous fission coincidences (cf. Table II).

$$
\begin{gathered}
\mathrm{SNR}_{\mathrm{S}}=\frac{S_{252 C f}}{S_{A m B e}+S_{60 C o}}=\frac{14966}{55933+196155}=0.06 \\
\mathrm{SNR}_{\mathrm{D}}=\frac{D_{252 C f}}{D_{A m B e}+D_{60 C o}}=\frac{4668}{3843+14381}=0.3 \\
\mathrm{SNR}_{\mathrm{T}}=\frac{T_{252 C f}}{T_{A m B e}+T_{60 C o}}=\frac{1175}{175+342}=2.3
\end{gathered}
$$

Plastic scintillators are however sensitive to neutron and gamma-ray multi-detector scattering (respectively elastic and Compton scattering), also known as scattering crosstalk. Therefore, compared to ${ }^{3} \mathrm{He}$ neutron counters that are practically insensitive to crosstalk, multiplicity analysis is not straightforward.

\section{B. Useful vs. background coincidence discrimination based on particle time of flight}

In an attempt to further improve SNR, we focus coincidence analysis on M2 and higher-order coincidences, and on the differences in times of flight between neutrons and gamma rays. The main idea is to represent all possible combinations of three coincident pulses in a two-dimensional time histogram, as shown in Fig. 4.The axes are the time intervals between the $1^{\text {st }}$ and $2^{\text {nd }}$ pulses $\left(\Delta_{2-1}\right.$ axis), and between the $2^{\text {nd }}$ and $3^{\text {rd }}$ pulses ( $\Delta_{3-2}$ axis). The regions of interest (ROI) on these $2 \mathrm{D}$ histograms are determined form the times of flight of gamma rays and neutrons between the sources and the detectors. The energy spectrum of ${ }^{252} \mathrm{Cf}$ neutrons typically follows a Watt/Maxwell law that spreads between approximately 0 and $10 \mathrm{MeV}$ [26]. The lowest detectable neutron energy with a 100 keVee threshold is about $700 \mathrm{keV} \mathrm{[27]} \mathrm{which} \mathrm{is} \mathrm{the} \mathrm{real} \mathrm{neutron} \mathrm{energy}$ corresponding to the scintillation light produced by $100 \mathrm{keV}$ electrons. This $700 \mathrm{keV}$ energy corresponds to a neutron speed of $1.15 \mathrm{~cm}^{-\mathrm{ns}^{-1}}$, while $2 \mathrm{MeV}$ (approximately the average energy of ${ }^{252} \mathrm{Cf}$ neutrons) and $10 \mathrm{MeV}$ neutrons (practically their maximum energy, even if higher energies are still possible but for few neutrons) travel at a speed of $2.0 \mathrm{~cm}^{-\mathrm{ns}^{-1}}$ and 4.3 $\mathrm{cm} . \mathrm{ns}^{-1}$, respectively. On the other hand, gamma rays travel much faster at a speed of $30 \mathrm{~cm} . \mathrm{ns}^{-1}$. With the $35 \mathrm{~cm}$ source-todetector radial distance, the time of flight of detected neutrons is thus approximately in the range [8-30] ns, while gamma rays are grouped within $1 \mathrm{~ns}$ interval. In reality, the length of the used detectors (cf. Fig. 1) enlarges the span of the source-todetector distances. Taken together with the time resolution of the detectors and data acquisition electronics (FWHM of about $4 \mathrm{~ns}$ ), it increases the range between pulse detection times.

Therefore, the $\gamma \gamma \gamma$ coincidences appear in the red triangles near the origin of the histograms, which extend to about $5 \mathrm{~ns}$ on each axis. The $\gamma \gamma \gamma$ region is densely populated in the case of the ${ }^{252} \mathrm{Cf}$ source because of the high prompt gamma ray multiplicity per spontaneous fission, which is close to 8 [26]. This region is also dense in the case of ${ }^{60} \mathrm{Co}$ because Compton scattering between detectors increases the order of original M1 coincidences between the $1.173 \mathrm{MeV}$ and $1.332 \mathrm{MeV}$ gamma rays. In addition, the ${ }^{60} \mathrm{Co}$ histogram also shows intense bands along the axes corresponding to accidental coincidences, when an uncorrelated pulse is detected with a correlated pair (noted "Acc $+\gamma \gamma$ " and " $\gamma \gamma+$ Acc" on Fig. 4).

Another noticeable region populated by $\gamma \gamma \mathrm{n}$ coincidences is visible in the ${ }^{252} \mathrm{Cf}$ and $\mathrm{AmBe}$ histograms near the $\Delta_{3-2}$ axis. It corresponds to a short time interval between the two first pulses, i.e. mainly $\gamma \gamma$ pairs, and to a longer delay between the $2^{\text {nd }}$ and $3^{\text {rd }}$ pulses of the triple coincidence, i.e. a $\gamma$ n pair.

The ${ }^{252} \mathrm{Cf}$ plot differs from the others by the region populated with $\gamma \mathrm{nn}$ coincidences due to spontaneous fissions. The latter are mostly triggered by a prompt gamma ray, followed by the detection of two prompt neutrons. The first one is in the [8-30] ns interval on $\Delta_{2-1}$ axis, and the second arrives up to $30 \mathrm{~ns}$ later, in the [0-30] ns range on $\Delta_{3-2}$ axis. The number of counts in this region of interest (ROI in red in Fig. 4) are given in Table III. The SNR is 5.1 with this new approach, which is significantly better than the SNR of 2.3 obtained in (3) with the shift register traditional method, which illustrates the potential interest of a coincidence selection based on particle times of flight. However, more work is needed to confirm this promising result, especially by performing acquisition with more intense sources measured simultaneously, to highlight the limits in terms of accidental coincidences. Moreover, measurements of these sources placed in mock-up drums filled with organic and metallic matrices (simulating technological wastes) have to be carried out, in order to assess detection sensitivity of high-order coincidences and the impact of gamma and neutron scattering on the contrast between the regions of interest of Fig. 4. 

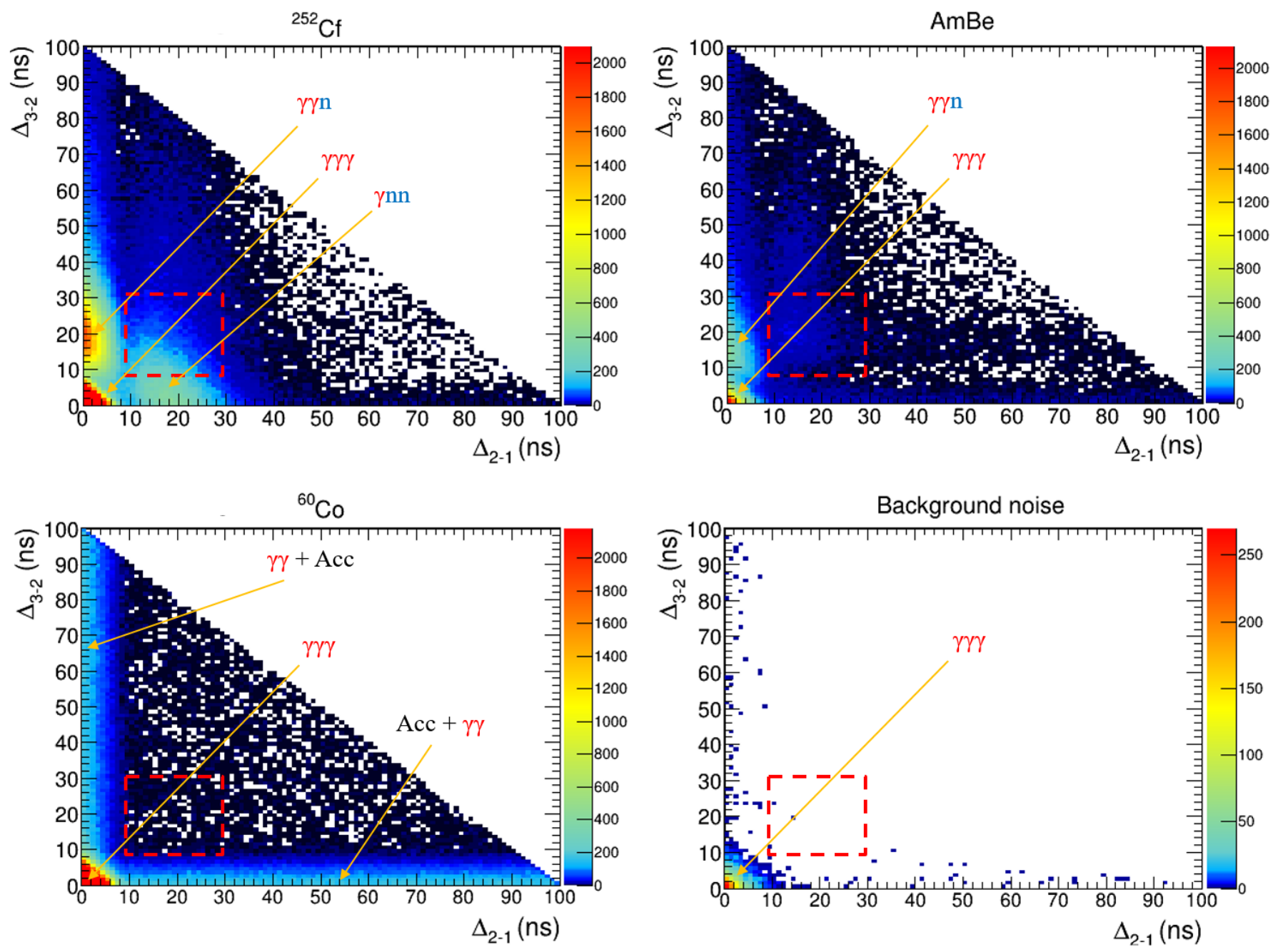

Fig. 4. Experimental two dimensional time histograms of all combinations of three coincident pulses in the measurements with ${ }^{252} \mathrm{Cf}$ (top left), AmBe (top right), ${ }^{60} \mathrm{Co}$ (bottom left) and background noise (bottom right). The color scale of ${ }^{252} \mathrm{Cf}$ and ${ }^{60} \mathrm{Co}$ plots is adjusted to that of AmBe (i.e. with a maximum near 2000 counts/bin). The ROI selected to provide best counting for the ${ }^{252} \mathrm{Cf}$ spontaneous fission coincidences while minimizing the parasitic ones is indicated, on each plot, by the red dashed-line rectangle ( $10 \mathrm{~ns} \leq \Delta_{2-1} \leq 30 \mathrm{~ns}$ and $\left.10 \mathrm{~ns} \leq \Delta_{3-2} \leq 30 \mathrm{~ns}\right)$.

TABLE III

NUMBER OF COUNTS IN THE REGION OF INTEREST OF FIG. 3 (RED RECTANGLES). SNR IS DEFINED BY THE RATIO OF THE COUNTS OBTAINED WITH ${ }^{252} \mathrm{CF}$, OVER COUNTS OBTAINED WITH OTHER SOURCES

\begin{tabular}{cc}
\hline Radioactive source & Counts in ROI \\
\hline${ }^{252} \mathrm{Cf}\left(3.6 \times 10^{4} \mathrm{n} \cdot \mathrm{s}^{-1}\right)$ & 28767 \\
$\mathrm{AmBe}\left(2.3 \times 10^{5} \mathrm{n} . \mathrm{s}^{-1}\right)$ & 4698 \\
${ }^{60} \mathrm{Co}(882 \mathrm{kBq})$ & 951 \\
Background noise & 3 \\
SNR & 5.1
\end{tabular}

\section{CONCLUSION}

The present work has shown that a discrimination between spontaneous-fission and background noise events of different origins can be achieved even with basic plastic scintillators without PSD capability, based on the time-of-flight differences of the detected neutrons and gamma rays. The preliminary oneby-one measurements performed with correlated neutron and gamma-ray sources $\left({ }^{252} \mathrm{Cf}, \mathrm{AmBe}\right.$, and ${ }^{60} \mathrm{Co}$ separate acquisitions) have evidenced that the SNR (signal-to-noise ratio) with the classical shift register method can be improved by sorting triple coincidences in a two-dimensional histogram of time intervals between the $2^{\text {nd }}$ and $1^{\text {st }}$ pulses (X axis) and between the $3^{\text {rd }}$ and $2^{\text {nd }}$ pulses ( $\mathrm{Y}$ axis). Contrary to PSD detectors that allow gamma rejection (after neutron-gamma discrimination), gamma pulses participate here to the useful signal, the ROI (region of interest) of $\gamma \mathrm{nn}$ coincidences leading to the best contrast (best SNR) between useful coincidences (spontaneous fissions of ${ }^{252} \mathrm{Cf}$ ) and parasitic ones $\left({ }^{60} \mathrm{Co}\right.$ and AmBe).

Future work will focus on scattering crosstalk rejection to further improve SNR. MCNPX-PoliMi simulations will also be implemented and validated with experimental data, in order to get better insight into the origin of the detected triple coincidences ( $\gamma \gamma \gamma, \gamma \mathrm{nn}, \gamma \gamma \mathrm{n}$, but also the difficult-to-observe nnn coincidences) and to optimize scattering crosstalk rejection.

On the other hand, as the present study addressed one-by-one measurements of neutron and gamma-ray sources (separate acquisitions), we will focus on high count rate measurements with all the sources together, and more specifically on the subtraction of accidental coincidences.

The number of detectors in the array will also be doubled, with 16 large PVT scintillators $(10 \mathrm{~cm} \times 10 \mathrm{~cm} \times 100 \mathrm{~cm})$ and 
a new high-count-rate FASTER electronics will be used [28]. The performances of this upgraded setup will be assessed with $\approx 100 \mathrm{~L}$ mock-up drums filled with organic or metallic matrices, to simulated realistic technological waste, in which the same sources but also plutonium samples will be placed in different positions. A $5 \mathrm{~cm}$ thick lead shield will also be used to limit the influence of plutonium gamma rays, and that of other gamma emitters like ${ }^{137} \mathrm{Cs}$ or ${ }^{60} \mathrm{Co}$. In this way, the discrimination between useful and background coincidences will be tested in realistic conditions, i.e. with significant neutron and gamma attenuation in the matrices, and with time-of-flight spreads due to neutron and gamma scattering. The assessment of measurement uncertainties due to matrix attenuation and source position will be of first importance in view of future developments towards an industrial application.

\section{ACKNOWLEDGMENT}

This work was supported by the CEA R\&D program for radioactive waste characterization.

\section{REFERENCES}

[1] N. Ensslin, "Principles of Neutron Coincidence Counting", Passive Nondestructive Analysis of Nuclear Materials, Los Alamos National Laboratory, chapter 16, 457-491.

[2] R.Kouzes et al. "Neutron detection alternatives to $3 \mathrm{He}$ for national security applications", Nucl. Instrum. Meth. A 623, 1035-1045 (2010)

[3] D. Henzlova et al. "Current Status of 3 He Alternatives Technologies for Nuclear Safeguards” LA-UR-15-21201 Ver. 3, PNNL-24307, July 2015.

[4] B. Simony, C. Deyglun, B. Pérot, C. Carasco, N. Saurel, S. Colas, J. Collot, "Cross-talk characterization in passive neutron coincidence counting of radioactive waste drums with plastic scintillators", IEEE Transactions on Nuclear Science, Vol. 63, No. 3, June 2016, 1513-1519.

[5] B. Simony, B. Pérot, C. Carasco, F. Jallu, N. Saurel, S. Colas, P. Girones, J. Collot, "Passive neutron coincidence counting with plastic scintillators for the characterization of radioactive waste drums", IEEE Transactions on Nuclear Science, Vol. 64, No. 10 (2017) 2719-2724.

[6] V. Bottau, L. Tondut, P.-G. Allinei, B. Pérot, C. Eleon, C. Carasco, R. De Stefano, G. Faussier, "High Resolution Gamma Spectrometry of Plutonium Bearing Waste Drum With High-Energy Reaction-Induced Gamma Rays" IEEE Transactions on Nuclear Science, Vol. 67, No. 4 (2020) 575-584.

[7] S. Li, S. Qiu, Q. Zhang, Y. Huo, H. Lin, "Fast-neutron multiplicity analysis based on liquid scintillation", Applied Radiation and Isotopes 110, 53-58 (2016).

[8] T. H. Shin, M. Y. Yua, M. J. Marcath, D. L. Chichester, I. Pázsit, A. Di Fulvio, S. D. Clarke, S. A. Pozzi, "Neutron Multiplicity Counting Moments for Fissile Mass Estimation in Scatter-Based Neutron Detection Systems", Nuclear Science and Engineering, 188:3, 246-249.

[9] R. Sarwar, V. Astromskas, C. H. Zimmerman, G. Nutter, A. T. Simone, S. Croft, M. J. Joyce, “An event-triggered coincidence algorithm for fast-neutron multiplicity assay corrected for cross-talk and photon breakthrough", Nucl. Instrum. Meth. A 903, 152-161 (2018).

[10] M. J. Joyce, R. Sarwar, V. Astromskas, A. Chebboubi, S. Croft, O. Litaize, R. Vogt, C. H. Zimmerman, "High-order angular correlation of californium-252 fission neutrons and the effect of detector cross-talk", Nucl. Instrum. Meth. A 954, 161866 (2020).

[11] N. Zaitseva et al. "Plastic scintillators with efficient neutron/gamma pulse shape discrimination", Nuclear Instruments and Methods in Physics Research A 668 (2012) 88-93.

[12] P. Roy, K. Banerjee, A. K. Saha, C. Bhattacharya, J. K. Meena, P. Bhaskar, S. Mukhopadhyay, S. Bhattacharya, "Detailed investigation on the possibility of using EJ-299-33A plastic scintillator for fast neutron spectroscopy in large scale experiments", Nucl. Instrum. Meth. A 911, 198-202 (2018).

[13] N. P. Zaitseva, A. M. Glenn, A. N. Mabe, M. L. Carman, C. R. hurlbut, J. W. Inman, S. A. Payne, "Recent developments in plastic scintillators with pulse shape discrimination", Nucl. Instrum. Meth. A 889, 97-104 (2018).

[14] M. P. Taggart, P. J. Sellin, "Comparison of the pulse shape discrimination performance of plastic scintillators coupled to a SiPM", Nucl. Instrum. Meth. A 908, 148-154 (2018).

[15] M. Ellis, C. Tintori, P. Schotanus, K. Duroe, P. A. Kendall, G. Mini, "The Effect of Detector Geometry on EJ-309 Pulse Shape Discrimination Performance", IEEE Nuclear Science Symposium Conference Record, 2013.

[16] C. Payne, P. J. Sellin, M. Ellis, K. Duroe, A. Jones, M. Joyce, G. Randall, R. Speller, "Neutron/gamma pulse shape discrimination in EJ299-34 at high flux", IEEE Nuclear Science Symposium Conference Record, 2015

[17] M. G. Paff, S. D. Clarke, S. A. Pozzi, "Organic liquid scintillation detector shape and volume impact on radiation portal monitors", Nucl. Instrum. Meth. A 825, 31-39 (2016).

[18] Banerjee, K. et al. "Variation of neutron detection characteristics with dimension of BC501A neutron detector". Nucl. Instrum. Meth. A 608, 440-446 (2009)

[19] CAEN Electronic Instrumentation, CAEN DT5730 (https://www.caen.it/products/compass/), accessed 28/07/2021

[20] CoMPASS, Multiparametric DAQ Software for Physics Applications (https://www.caen.it/products/dt5730/), accessed 28/07/2021

[21] N. Ensslin, "The Origin of Gamma Rays", Passive Nondestructive Analysis of Nuclear Materials, Los Alamos National Laboratory, chapter 1, pp. 1-25.

[22] ROOT Data Analysis Framework, CERN, https://root.cern.ch, accessed 28/07/2021.

[23] K. Boehnel "Determination of plutonium in nuclear fuels using the neutron coincidence method", March 1978.

[24] N. Ensslin, W. C. Harker, M. S. Krick, D.G. Langner, M. M. Pickrell, J. E. Stewart, "Application Guide to Neutron Multiplicity Counting”, LA13422-M, November 1998

[25] D. Henzlova, H. O. Menlove, S. Croft, A. Favalli, P. Santi, "The impact of gate width setting and gate utilization factors on plutonium assay in passive correlated neutron counting", Nucl. Instrum. Meth. A 797, 144152 (2015).

[26] N. Ensslin, "Principles of Neutron Coincidence Counting", Passive Nondestructive Analysis of Nuclear Materials, Los Alamos National Laboratory, chapter 11, pp. 337-354.

[27] S. A. Pozzi, J. A. Mullens, J. T. Mihalczo, "Analysis of neutron and photon detection position for the calibration of plastic (BC-420) and liquid (BD-501) scintillators", Nucl. Instrum. Meth. A 524, 92-101 (2004).

[28] FASTER, Fast Acquisition SysTem for nuclEar Research, https://faster.in2p3.fr, accessed 28/07/2021. 